\title{
THE DYNAMIC LOADING IN LONGITUDINAL AND TRANSVERSE REINFORCEMENT AT INSTANT EMERGENCE OF THE SPATIAL CRACK IN REINFORCED CONCRETE ELEMENT UNDER THE ACTION OF A TORSION WITH BENDING
}

\author{
Aleksey Demyanov* \\ South-West State University, Kursk, Russia \\ Vladimir Ivanovich Kolchunov \\ South-West State University, Kursk, Russia
}

It is proposed the computational model of the dynamic loading in longitudinal and transverse reinforcement at instant emergence of the spatial crack in reinforced concrete constructions under the action of torsion with bending. The dependences have been obtained for the determination of stresses in stretched reinforcement, vertical and horizontal reinforcement stirrups in cross section with a crack.

Key words: Bilinear surface, Dynamic loading, Emergence of crack, Torsion with bending,

Reinforced concrete structures

\section{INTRODUCTION}

The task of studying dynamic loading under the emergence of crack in reinforced concrete elements is currently poorly understood [01-04]. In particular, it remains open for the complex stressed state of the reinforced concrete structure under the action of torsion with bending [05-
08]. The present of the paper is devoted to solving this problem.

To determine the value of the dynamic force, let's turn to the bilinear surface obtained earlier in article [09]. It describes the shape of the spatial crack of the reinforced concrete element (Figure 1).

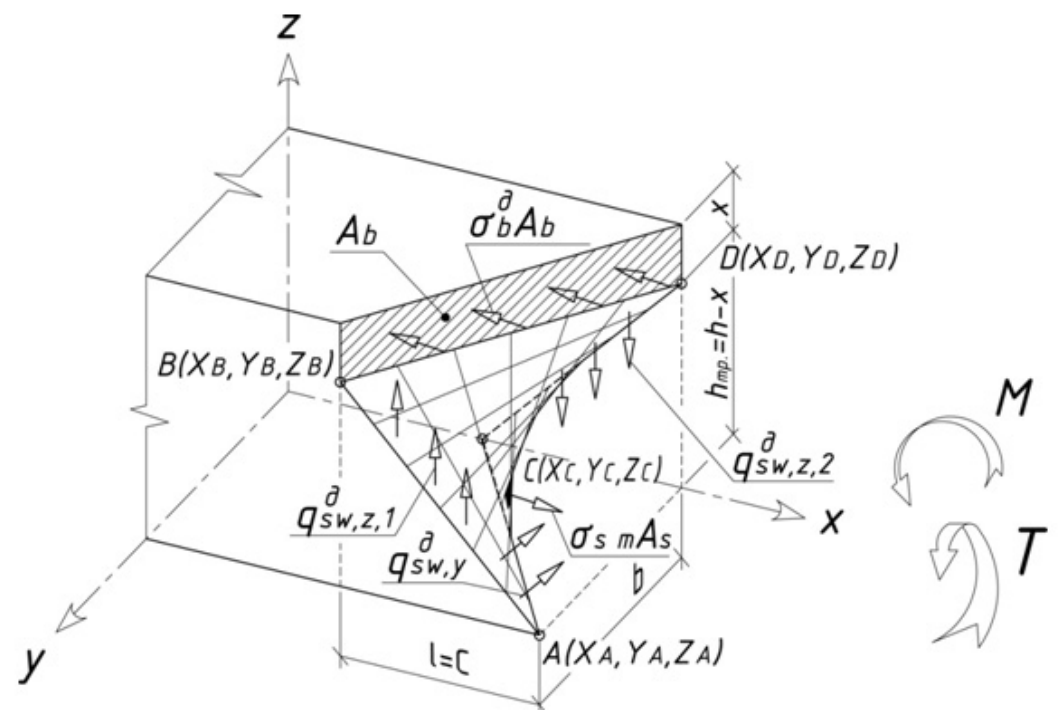

Figure 1: The general view of emergence crack and mathematical model of its bilinear surface in a reinforced concrete element under the action torsion with bending 
Consider this surface, dividing it into elementary

tion model. squares (Figure 2, a, b) In the proposed calcula-
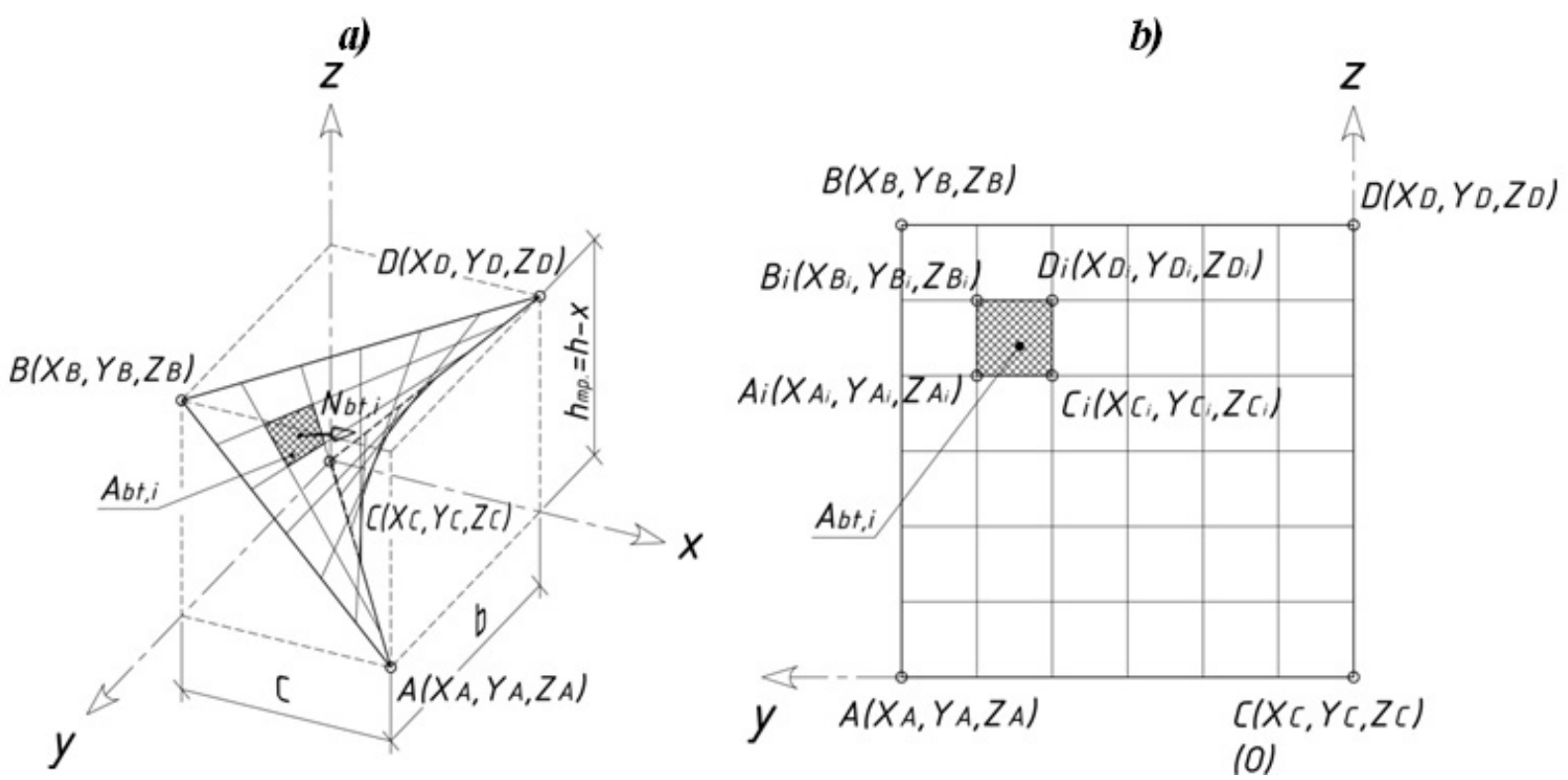

Figure 2: The model of the bilinear surface of the spatial crack in reinforced concrete element, divided into elementary squares (a) and its view in the plane YOZ (b)

\section{RESEARCHES AND RESULTS}

Mentally, we divide this surface into squares with corner points $\mathrm{Ai}, \mathrm{Bi}, \mathrm{Ci}, \mathrm{Di}$, the total number of which, within the cross-sectional area, is $\mathrm{m}$. At a point located at the intersection of the diagonals of each square, the force Nbt,i, is applied along its normal to its surface (see Figure 2 (a)), which corresponds to the ultimate tensile strength of concrete in the area of this square. It is determined by the formula:

$$
N_{b t, i}=R_{b t} \cdot A_{b t, i} \text {, }
$$

where $i=1 \div m ; A b t, i-$ is the area of an elementary square, which can be easily determined from the coordinates of the corner points of the square AiBiCiDi. Note that the coordinates of the corner points can be easily found by the method given in [09].
The only unknown parameter is the orientation of the force Nbt, in space of this case. We single out one of the elementary squares AiBiCiDi and consider it more detail (Figure 2, Figure 3).

It has drawn through the point Wi [xwi, ywi, zwi] the forces $\mathrm{Nbt}$,i, the lines $\mathrm{HiLi}$ and $\mathrm{MiNi}$, which divide the lines $\mathrm{AiBi}(\mathrm{CiDi})$ and $\mathrm{AiCi}(\mathrm{BiDi})$ in half. The coordinates of the points $\mathrm{Hi}, \mathrm{Li}, \mathrm{Mi}, \mathrm{Ni}$ are defined similarly to the corner points of the square $\mathrm{AiBiCiDi}$.

The equation of a bilinear surface in parametric form is known, we enter into it, the value of the corner points of the reinforced concrete structure cross-section-points $A, B, C, D$ is inscribed in it. The equation of a bilinear surface is concretized with respect to the given cross-section.

$$
\begin{aligned}
{\left[x_{k} ; y_{k} ; z_{k}\right] } & =\left[x_{A} ; y_{A} ; z_{A}\right] \cdot\left(1-u_{k}\right) \cdot\left(1-w_{k}\right)+\left[x_{B} ; y_{B} ; z_{B}\right] \cdot\left(1-u_{k}\right) \cdot w_{k}+ \\
& +\left[x_{C} ; y_{C} ; z_{C}\right] \cdot u_{k} \cdot\left(1-w_{k}\right)+\left[x_{D} ; y_{D} ; z_{D}\right] \cdot u_{k} \cdot w_{k} .
\end{aligned}
$$

The bilinear surface is split into the corresponding squares and the coordinates of any point of the square are calculated from the specific equation of the bilinear surface. In this case, the step of splitting into squares $u$ and $w$ is given, that is, the values of uk and $w k$ in the parametric equation (2) are known. Then, it is possible to determine the coordinates of the three points of any small square (AiBiCiDi, Figure 2) falling into a given grid, for example, points $\mathrm{M}, \mathrm{H}$ and $\mathrm{W}$ : 


$$
\begin{aligned}
{\left[x_{M} ; y_{M} ; z_{M}\right] } & =\left[x_{A} ; y_{A} ; z_{A}\right] \cdot\left(1-u_{M}\right) \cdot\left(1-w_{M}\right)+\left[x_{B} ; y_{B} ; z_{B}\right] \cdot\left(1-u_{M}\right) \cdot w_{M}+ \\
& +\left[x_{C} ; y_{C} ; z_{C}\right] \cdot u_{M} \cdot\left(1-w_{M}\right)+\left[x_{D} ; y_{D} ; z_{D}\right] \cdot u_{M} \cdot w_{M} ; \\
{\left[x_{H} ; y_{H} ; z_{H}\right] } & =\left[x_{A} ; y_{A} ; z_{A}\right] \cdot\left(1-u_{H}\right) \cdot\left(1-w_{H}\right)+\left[x_{B} ; y_{B} ; z_{B}\right] \cdot\left(1-u_{H}\right) \cdot w_{H}+ \\
& +\left[x_{C} ; y_{C} ; z_{C}\right] \cdot u_{H} \cdot\left(1-w_{H}\right)+\left[x_{D} ; y_{D} ; z_{D}\right] \cdot u_{H} \cdot w_{H} ; \\
{\left[x_{W} ; y_{W} ; z_{W}\right] } & =\left[x_{A} ; y_{A} ; z_{A}\right] \cdot\left(1-u_{W}\right) \cdot\left(1-w_{W}\right)+\left[x_{B} ; y_{B} ; z_{B}\right] \cdot\left(1-u_{W}\right) \cdot w_{W}+ \\
& +\left[x_{C} ; y_{C} ; z_{C}\right] \cdot u_{W} \cdot\left(1-w_{W}\right)+\left[x_{D} ; y_{D} ; z_{D}\right] \cdot u_{W} \cdot w_{W} ;
\end{aligned}
$$

If the coordinates of the three points $M\left(x_{m} ; y_{m} ;\right.$ $\left.z_{m}\right), H\left(x_{H} ; y_{H} ; z_{H}\right)$ and $W\left(x_{w} ; y_{w} ; z_{w}\right)$ are known, the equation of the surface for a small square is written in coordinate form:

$$
\left|\begin{array}{ccc}
x-x_{M} & y-y_{M} & z-z_{M} \\
x_{H}-x_{M} & y_{H}-y_{M} & z_{H}-z_{M} \\
x_{W}-x_{M} & y_{W}-y_{M} & z_{W}-z_{M}
\end{array}\right|=0 .
$$

After expanding the determinant of equation (6), we obtain the equation of the surface for a small square in the following form:

$$
A x+B y+C z+D=0 \text {, }
$$

where the coefficients $A, B, C, D$ are determined from the disclosure of the determinant. Then, the equation of the normal to the found surface at a point $\mathrm{W}$ in the parametric form is written down. In this case, the condition of perpendicularity of the normal to the plane of the small square is taken into account:

$$
\frac{A}{a_{1}}=\frac{B}{a_{2}}=\frac{C}{a_{3}} \text {. }
$$

In equation (8), the parameters $A, B, C, D$ are known, and the values of unknowns $a_{1}, a_{2}$ are determined from using these proportions:

$\frac{A}{a_{1}}=\frac{C}{a_{3}} \Rightarrow a_{1}=\frac{A \cdot a_{3}}{C} ; \quad \frac{B}{a_{2}}=\frac{C}{a_{3}} \Rightarrow a_{2}=\frac{B \cdot a_{3}}{C}$.

The known relation between the direction cosines of the normal is used to determine the unknown parameter $\mathrm{a}_{3}$ by next formula:

where

$$
l^{2}+m^{2}+n^{2}=1
$$

$l=\frac{a_{1}}{d} ; m=\frac{a_{2}}{d} ; n=\frac{a_{3}}{d} ; d=\sqrt{\left(a_{1}\right)^{2}+\left(a_{2}\right)^{2}+\left(a_{3}\right)^{2}}$.

Then, it is divided each term of the equation (10) to $\mathrm{n}^{2}$. Then, we will have:

$$
\left(\frac{l}{n}\right)^{2}+\left(\frac{m}{n}\right)^{2}+1=\left(\frac{1}{n}\right)^{2} .
$$

Transform equation (12), using relations (9):

$$
\begin{aligned}
& \frac{l}{n}=\frac{a_{1}}{d} \cdot \frac{d}{a_{2}}=\frac{a_{1}}{a_{2}}=\frac{A \cdot a_{3}}{C} \cdot \frac{C}{B \cdot a_{3}}=\frac{A}{B} ; \\
& \frac{m}{n}=\frac{a_{2}}{d} \cdot \frac{d}{a_{3}}=\frac{a_{2}}{a_{3}}=\frac{B \cdot a_{3}}{C} \cdot \frac{1}{a_{3}}=\frac{B}{C} .
\end{aligned}
$$

Substituting expressions (13) and (14) into equation (12), we have:

$$
\left(\frac{A}{B}\right)^{2}+\left(\frac{B}{C}\right)^{2}+1=\left(\frac{1}{n}\right)^{2} \text {. }
$$

Then, carrying out the corresponding algebraic transformations, we obtain an expression for the direction cosine $n$ :

$$
n= \pm \sqrt{\frac{\frac{1}{n^{2}}=\frac{A^{2}}{B^{2}}+\frac{B^{2}}{C^{2}}+1 ;}{B^{2} \cdot C^{2}} \cdot C^{2}+B^{2} \cdot B^{2}+B^{2} \cdot C} .
$$

Now, using relations (9) and the resulting expression (15), after algebraic transformations we obtain:

$$
\frac{l}{n}=\frac{A}{B} \Rightarrow l=\frac{A \cdot n}{B}= \pm \frac{A}{B} \cdot \sqrt{\frac{B^{2} \cdot C^{2}}{A^{2} \cdot C^{2}+B^{2} \cdot B^{2}+B^{2} \cdot C}} ;
$$

$\frac{m}{n}=\frac{B}{C} \Rightarrow m=\frac{B \cdot n}{C}= \pm \frac{B}{C} \cdot \sqrt{\frac{B^{2} \cdot C^{2}}{A^{2} \cdot C^{2}+B^{2} \cdot B^{2}+B^{2} \cdot C}}$

As a result, it is obtained the dependences (15) (17) for determining the direction cosines to the normal, Figure 3. 


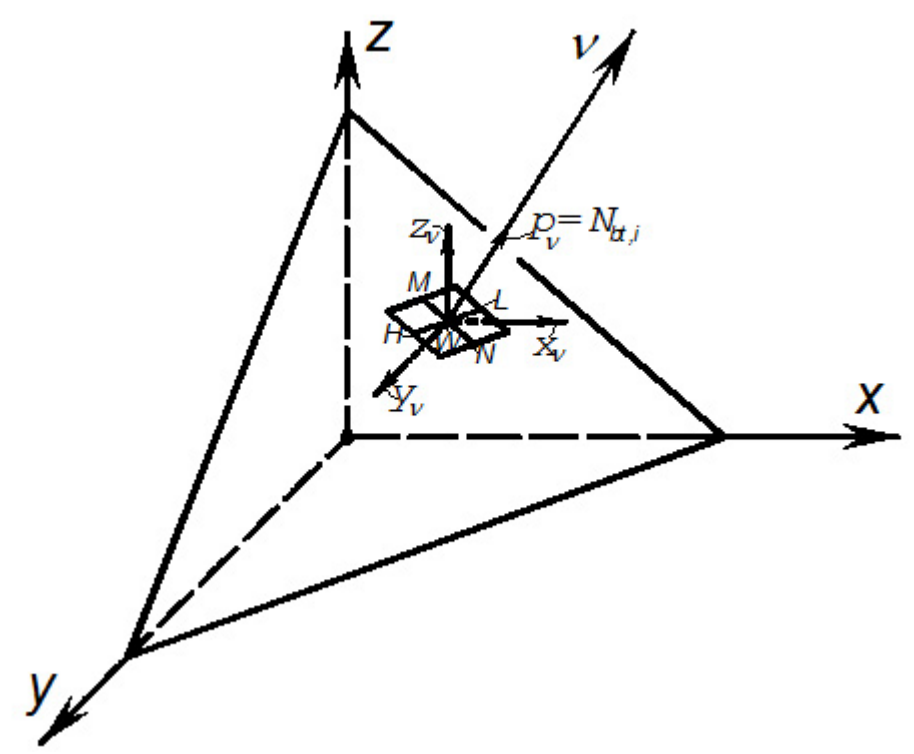

Figure 3: The normal to the surface of a small square at the point $W$ at the intersection of the lines $M N$ and $H L$ and the decomposition of the normal force PV

The equal component of the effort on the inclined area $\mathrm{xv}, \mathrm{yv}, \mathrm{zv}$ (Figure 3 ) is the total effort at this site and is defined as the geometric sum of the components:

$P_{v}=N_{b t, i}=\sqrt{X_{v}^{2}+Y_{v}^{2}+Z_{v}^{2}}$,

where

$$
X_{v}=N_{b t, x} \cdot l ; \quad Y_{v}=N_{b t, y} \cdot m ; \quad Z_{v}=N_{b t, z} \cdot n
$$

and $\cos (\mathrm{x}, \mathrm{v})=\mathrm{I}, \cos (\mathrm{y}, \mathrm{v})=\mathrm{m}$ and $\cos (\mathrm{z}, \mathrm{v})=\mathrm{n}$ are calculated by formulas (15)-(17).

Return to Figure 1 and consider which dynamic loadings occur in the reinforcement element in the deplanated section with a spatial crack. To solve this problem, we turn to the article [10]. The task of introducing into account the efforts of dynamic loadings preloading of reinforcement is reduced to a number of corrections to the equation of internal forces already obtained within the present work in the spatial section of the element. The calculation algorithm is the following.

At the first stage, a full calculation is carried out according to the recommendations given in [10]. The second stage is based on the obtained data on the projection length "c" of the spatial crack on the $X$ axis and the height of the compressed concrete zone $x b$ of the deplanated section with a spatial crack, determine the coordinates of the angular points of the bilinear surface of the spatial crack and build it.

In the third stage of the calculation, the bilinear surface is divided into $m$ elementary squares and, in accordance with the recommendations given above, the components of the forces Nbt,i are determined for each square along the $X, Y$ and $Z$ axes.

\section{CONCLUSIONS}

To determine the dynamic loading in longitudinal and transverse reinforcement, it is used the proposition set forth in $[01,02,04]$, at the accordance with which:

$$
\sigma_{s, n-1}^{\partial}=2 \cdot \sigma_{s, n-1}^{c}-\sigma_{s, n}^{c}
$$

where $\sigma_{s, n-1}^{\partial}$ - the stresses in the reinforcement, takıng into account the dynamic loading from the instantaneous emergence of a spatial crack; $\sigma_{s, n}^{c}$-the stresses in the stretched working reinforcement in the cracked section that would have arisen in this system if the n-system transition to the (n-1) system was not due to the instantaneous destruction of the stretched part of the concrete in the cross section $\mathrm{k}$, but by its slow forced unloading from some value of the limiting stress, perceived by this part of the section to zero; the stresses in the reinforcement obtained during the static calculation of the system at the stage preceding the moment of emergence of a spatial crack.

Specifying this formula, applied to the longitudinal reinforcement, taking into account the parameters given in this paper, we obtain: 


$$
\sigma_{s, n-1}^{\partial}=2 \cdot \sigma_{s, n-1}-2 \cdot \alpha R_{b t} \cdot l \text {. }
$$

Specifying this formula, applied to the transverse reinforcement, taking into account the parameters given in this paper, we obtain:

$$
\sigma_{s w, n-1}^{\partial}=2 \cdot \sigma_{s w, n-1}-2 \alpha R_{b t} \cdot \cos \alpha_{i} \text {. }
$$

where $\sigma_{s w, n-1}^{\partial} \quad-$ the stresses in the vertical or horizontal stirrups reinforcement, taking into account the dynamic loading from the instantaneous emergence of a spatial crack; $\sigma_{s w, n-1}$ the stresses in the vertical or horizontal stirrups reinforcement after the appearance of the spatial crack, obtained by the calculation of first stage; $2 a \mathrm{abt}$ - the stresses in the working armature in the stage preceding the moment of spatial crack emergence; the parameter takes values for vertical stirrups, but for horizontal stirrups reinforcement.

It will be assumed that the spatial crack crosses the reinforcing bar perpendicular to its longitudinal axis.

To determine the area of the reinforcing bar of the longitudinal working reinforcement (or vertical or horizontal stirrups) at the point where it intersects the spatial crack and the stresses arising in this section, consider the elementary square $\mathrm{AjBjCjDj}$. The side length value of the square is $0,9 \mathrm{dj}$ ( $\mathrm{dj}$ is the diameter of the jth reinforcing bar) and the intersection point of its diagonals coincides with the point of intersection of the spatial crack with the axis of the reinforcing bar (Figure 4).

a)

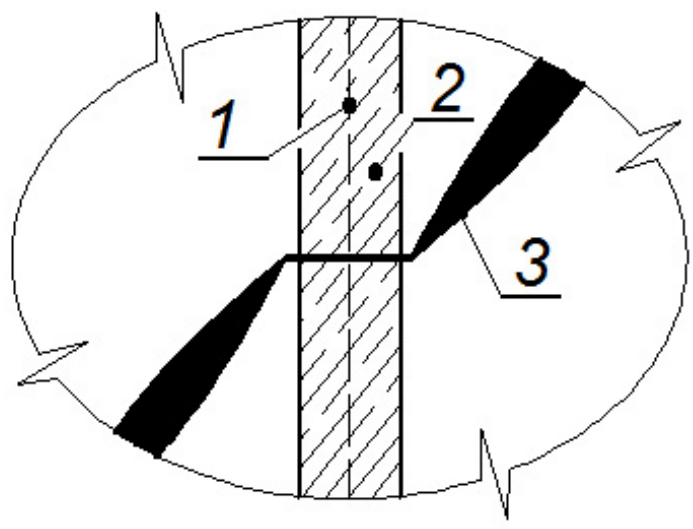

Note that in the YOZ plane of the cross-section for longitudinal reinforcement, in the XOZ plane of the cross-section for horizontal stirrups and in the XOY plane of the cross-section for vertical stirrups are circles (Figure 4, b), according to the accepted assumption.

The dynamic linear efforts (see Figure 1) in horizontal stirrups are defined as follows:

$$
q_{s w, y}^{\partial}=2 \cdot q_{s w, y}-\omega_{y} \cdot 2 \alpha R_{b t} \cdot m \text {, }
$$

where $q_{s w, y}^{\partial}$ corresponds to $q_{s w, y}$ from [10], obtained during the first stage of the calculation; - filling diagrams of the tensile stresses of the reinforcement in the direction of the $Y$ axis along the spatial crack, reduced to linear.

The dynamic linear efforts (see Figure 1) in vertical stirrups are defined as follows:

$$
q_{s w, z, 1}^{\partial}=2 \cdot q_{s w, z, 1}-\omega_{z} \cdot 2 \alpha R_{b t} \cdot n,
$$

where if ,then corresponds from [10], obtained during the first stage of the calculation; - filling diagrams of the tensile stresses of the reinforcement in the direction of the $\mathrm{Z}$ axis along the spatial crack, reduced to linear;

$$
q_{s w, z, 2}^{\partial}=2 \cdot q_{s w, z, 2}-\omega_{z} \cdot 2 \alpha R_{b t} \cdot n,
$$

where if ; then corresponds from [10], obtained during the first stage of the calculation.

b)

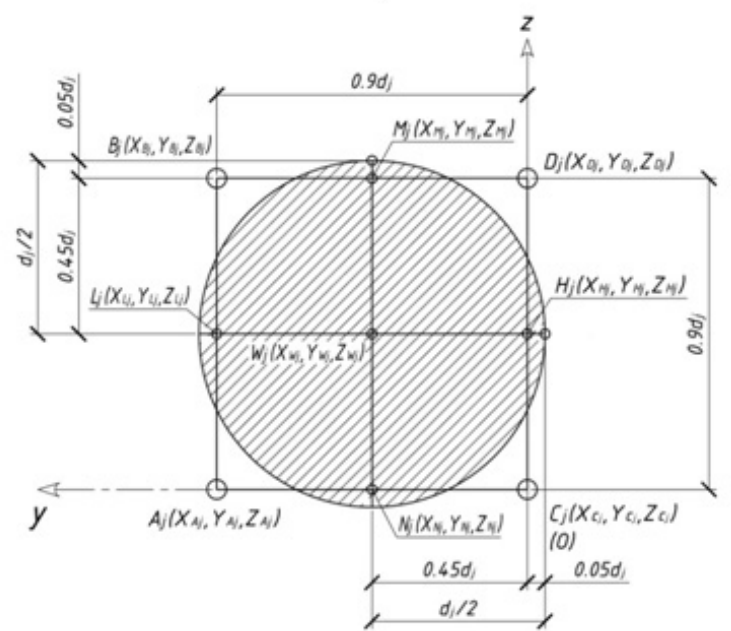

Figure 4: The fragment of the intersection of the vertical reinforcing stirrups (or horizontal stirrups or longitudinal working armature) by a spatial crack (a) and determination of theit area at the point where it intersects the spatial crack (b); 1 - the central axis of the vertical reinforcement bar; 2 - vertical reinforcement bar; 3- spatial crack 
The linear nagel effort in the reinforcement stirrups [11, 12], arising on the side faces of the reinforced concrete element (not shown in Fig. 1) - is determined by:

$$
q_{2,5 w}^{\partial}=2 \cdot q_{2,5 w}-\omega_{x} \cdot 2 \alpha R_{b t} \cdot l \text {, }
$$

where - filling the stress tensile stresses of the reinforcement in the direction of the $X$ axis along the spatial crack, reduced to linear.

As a result of the calculations, all the components of dynamic loading are obtained of the reinforcement from the instantaneous of a spatial crack emergence that occurs when the element is under the action of torsion with bending.

Thus, as a result of these studies, it is proposed a design model for the dynamic loading of reinforcement from the instantaneous formation of a spatial crack that occurs when a reinforced concrete structure is under torsion with bending and the dependences for determining stresses in stretched reinforcement, vertical and horizontal stirrups in a section with a crack are obtained.

\section{REFERENCES}

1) Geniev G. A., Kolchunov V. I., Klueva N. V. \& oth. (2004), The strength and deformability of reinforced concrete of constructions at beyond design impacts, ASV Publ., Moskow, 216 p. [In Russian]

2) Kolchunov V. I., Klueva N. V., Androsova N. B., Bukhtiyarova A. S. (2014). Vitality of buildings and structures under beyond design basis impacts. ASV Publ., Moskow, 208 p. [In Russian]

3) Emelyanov, S., Nemchinov, Y., Kolchunov, V., \& Yakovenko, I. (2016). Details of largepanel buildings seismic analysis. Enfoque UTE, 7(2), pp. 120 - 134. [In English].

4) Kolchunov VI.I., Yakovenko I.A. (2016). The calculation model of static and dynamic deformation reinforced concrete constructions in bending at the destruction moment of stretched concrete matrix. Scientific Journal "Transactions of Kremenchuk Mykhailo Ostrohradskyi National University". Vol. 3(98), part 1, pp. 56-62. [In Russian]

5) Kolchunov VI.I., Fedorov V.S., Pokusaev A.A. (2016). The calculation of the distance between spatial cracks and the width of their opening in reinforced concrete structures under torsion with bending. Housing construc- tion. Vol. 5, pp. 31-37. [In Russian]

6) Kolchunov VI.I., Salnikov A.S. (2016). The results of experimental studies of cracking of reinforced concrete structures in torsion with bending. Building and reconstruction. Vol. 6 (68), pp. 22-28. [In Russian]

7) Salnikov A.S., Klueva N. V., Kolchunov VI.I. (2016). The method for determining the minimum load and the coordinates of the spatial crack formation in reinforced concrete structures under torsion with bending. Industrial and Civil Engineering. Vol. 1, pp. 52-57. [In Russian]

8) Salnikov A., Kolchunov VI., Yakovenko I. (2015). The computational model of spatial formation of cracks in reinforced concrete constructions in torsion with bending // Applied Mechanics and Materials. Vols. 725726, pp. 784-789. [In English].

9) Kolchunov VI.I., Rypakov D.A. (2016). Modeling spatial cracks in concrete structures under torsion with bending. Structural Mechanics of Engineering Constructions and Buildings. Vol. 5, pp. 11-16. [In Russian]

10) Pokusaev A.A., Schavukina M.V., Kolchunov V.I. (2016). The calculation of the distance between spatial cracks and the width of their opening in reinforced concrete structures under torsion with bending (case 1). Structural Mechanics of Engineering Constructions and Buildings. Vol. 2, pp. 20-29. [In Russian]

11) Bondarenko, V. M. and Kolchunov, V. I. (2004), The computational model of a power resistance of reinforced concrete, ASV Publ., Moskow, 472 p. [In Russian]

12) Golishev, A. B. and Kolchunov, V. I. (2009), The resistance of reinforced concrete, Osnova Publ., Kiev, 432 p. [In Russian]

Paper sent to revision: 19.07.2017.

Paper ready for publication: 15.09.2017. 